\title{
Inflammation and autoimmunity: risk assessment and predictive approaches through the analytical armamentarium
}

THE HUMAN MICROBIOME DEMYSTIFIES CHRONIC INFLAMMATORY DISEASE, ALLOWING PREDICTION AND PREVENTION IN 'AUTOIMMUNE', CARDIOVASCULAR, DIABETIC, AND NEURODEGENERATIVE DISORDERS

Marshall TG

Murdoch University of Western Australia, Perth, Australia Autoimmunity Research Foundation, California, USA

The absence of a clear understanding of human chronicdisease, especially the absence of a clear pathogenesis, has hindered implementation of targeted predictive and preventive medicine. But the emerging sciences of metagenomics and proteomics have now clarified a mechanism whereby not just one or two discrete pathogens can drive the disease process, but where a very large number of viral, fungal and bacterial species, accumulating in the human body over the course of a lifetime, progressively weaken the human innate immune system. Homo sapiens is not a sterile compartment, but a super-organism harboring thousands of species of microbes in its cells and tissues. Those microbes which have evolved to persist inside nucleated and phagocytic cells can engender both genomic and proteomic interference within the host cell metabolism, precipitating the disease processes. Key components of this microbiota persist by reducing expression of, and by, the VDR Nuclear Receptor, which is at the heart of the human innate immune system. Only in Homo sapiens is the VDR responsible for expression of Cathelicidin, TLR2, TACO and a number of other endogenous anti-microbials. Animals have different antimicrobial defenses, and thus cannot properly model human chronic disease. Pathogens already known to persist by dysregulating the human VDR include EBV, Mycobacteria, Borrelia, Aspergillus and HIV. Antimicrobial therapy is difficult, as the diseased
VDR cannot be simply restored by endogenous metabolic processes, as it can in healthy individuals. Nor can antibiotics function in-vivo in the absence of a competent innate immune system. We have shown that the drug Olmesartan can be retargeted to function as a VDR agonist, allowing innate immunity to recognize the pathogens, and reverse the disease process. Recovery is very slow, as care must be exercised to ensure the resulting cytokine-storm does not become life-threatening. Over the last decade, we have implemented a worldwide clinical collaboration to demonstrate 'proof of concept' efficacy in hundreds of subjects with serious chronic disorders, ranging from schizophrenia and manic depression, through ALS and MS, to sarcoidosis and arthritis. It seems certain that the current epidemic of chronic disease can not only be controlled, it can be reversed by implementation of preventative measures aimed at minimizing the accumulation of pathogenic organisms into the human microbiome.

\section{FEATURES OF THE INNATE AND ADAPTIVE IMMUNITY IN PATIENTS WITH INTRACRANIAL INFECTIOUS AND INFLAMMATORY DISEASES Sautin $M^{1}$, Cherepakhina $N^{2}$, Bodrova $T^{3}$, Suchkov $S^{2}$ \\ ${ }^{1}$ Peoples' Friendship University of Russia, Moscow, Russia ${ }^{2}$ I.M. Sechenov First Moscow State Medical University, Moscow, Russia \\ ${ }^{3}$ Higher School of Economy, Moscow, Russia}

Introduction: Diagnosis, treatment, prognosis of intracranial suppurative complications after surgical interventions for diseases and injuries of the nervous system and inflammatory diseases of the upper respiratory tract is an important problem of pediatric surgery. Of great and special impor- 
tance is a clinical and immune-mediated post-infectious syndrome (CIPIS) to play a crucial role in the chronization of the acute infections and to contribute to the development of the disease-related complications.

Objectives: To study features of the immune responsiveness of patients with different clinical forms of intracranial infectious and inflammatory processes (ICIIP), to assess the clinical relevance of the data obtained and to create a model of the ICIIP immunopathogenesis and some of the typical forms of ICIIP associated with CIPIS in the pathogenesis of ICIIP.

Materials and methods: 88 serum samples of patients with ICIIP confirmed by imaging tests (CT, MRI) or during the surgery (brain abscesses - 55; dural empyema - 25; meningoencephalitis -8 ) and 29 clinically healthy donors have been studied. Cytometry was used for immunophenotyping of blood cell subsets; screening of cytokines, for anti-tissue, anti-organ and antimicrobial antibodies (Abs) as well as for B7-H1 biomarker by ELISA and cytometry; identification of infectious pathogens was done by culture and PCR.

Results: Two major forms of ICIIP-associated CIPIS have been identified and confirmed, namely,

(1) postinfectious secondary immunodeficiency syndrome (PISIS) - $92 \%$ of the total number of patients (including aggressive subtypes: $38 \%$ and moderate ones: $54 \%$ );

(2) PISIS associated with autoimmune syndrome (PISIDAS) - around $10 \%$ of patients.

ICIIP is characterized by the development of clinically expressed subtypes of PISIS (38\%), moderate types of PISIS (54\%), PISIDAS (10\%). Specific features of either of two syndromes have been established, namely, for:(I) innate immunity and (II) effector link of adaptive immunity and regulatory mechanisms of the immunity as a whole.

Information obtained from the studies has given a way to define two new classes of criteria to be implemented into clinical and preclinical practice. The first class would include microbiome-based criteria to monitor spectrum and the localization of the microbial gene pool and serologic profile anti-microbial Abs. The second one would include immune-based criteria to predict risks of transformation (predictive factor) of acute forms of ICIIP into the chronic ones and development of complications.

This study sets out the potential molecular and cellular targets to be used for developing new drugs to treat CIPIS and ICIIP and to predict the chronization as well as the efficacy of therapeutic strategies in clinical and preclinical stages of the disorder as the task of personalized medicine.
Further research is required to understand how CIPIS is being developed in the preclinical stages and to develop methods to perform clinically and pre-clinically (preventive) applicable therapies for ICIIP.

POST-INFECTIOUS CLINICAL-IMMUNOLOGICAL SYNDROME AND ITS ROLE IN THE STATE-OFTHE-ART MODEL OF IMMUNOPATHOGENESIS OF CHRONIC INFECTIOUS DISEASES

Agirov $\mathrm{MM}^{2,5}$, Tabaksoeva $\mathrm{JA}^{2}$, Akhmedilova $K A^{2}$, Cherepakhina $N E^{1,3}$, Shogenov $\mathrm{ZS}^{2}$, Bodrova $T A^{4}$, Poteshkina $N G^{2}$, Suchkov SV ${ }^{1,2}$

${ }^{1}$ I.M. Sechenov First Moscow State Medical University (FMSMU), Moscow, Russia

${ }^{2}$ Russian State Medical University (RSMU), Moscow, Russia

${ }^{3}$ Federal Agency of Medical and Biological Problems under the Government of the Russian Federation, Moscow, Russia ${ }^{4}$ Department of Economy of Health Services, The Higher School of Economy, Moscow, Russia

${ }^{5}$ University of World Politics and Law, Faculty of Economy of Medical Technologies, Moscow, Russia

The Post-infectious clinical-immunological syndrome (PICIS) variants include:

(1) Post-infectious secondary immunodeficiency syndrome (PIFSI);

(2) Post-infectious autoimmune syndrome (PIFA);

(3) PIFAS coupled with PIFA (PIFASID).

The incidence of the above listed syndromes in patients with CID/CDIN differs statistically depending on the form of the primary infectious pathology and the stage of the destructive inflammatory process in target organs or tissues. For example, the initial (including preclinical) stages are usually concomitant with the predominant $>50 \%$ ) formation of PIFSI, whereas the share of PIFA and PIFASID does not exceed $20 \%$.

This situation changes with further development and chronization of the infectious process and its transformation into the clinical phase, viz., and the share of autoimmune syndromes increases substantially. In the intermediate stages of chronization, the share of PIFA approximates $50 \%$, while the share of PIFASID in the final stages reaches $60 \%$.

The frequency of PIFSI detection decreases appreciably with the increase in the duration of the disease and deterioration of cicatrical sclerotic lesions (to 25-40\%); that in the stage of pronounced substitution of the parenchyma with connective tissue drops down to $10-15 \%$. The correlation between the stage of the disease and the form of PICIS is characterized by the presence of an 
additional component, namely, the form and the clinical course of the disease. There are some examples related to:

(1) Clinical form of the disease. In patients with primary pyelonephritis (PPN) and infectious myocarditis (IM), PIFSI occurs in $75 \%$ of cases, while in patients with secondary pyeolnephritis (SPN) and autoimmune myocarditis (AIM) the PIFSI ratio drops down to $25 \%$ giving way to autoaggressive symptomatology, that is, the incidence of PIFA and PIFASID increases (up to 60 and $85 \%$, respectively).

(2) Period of disease: In early (less than 3 months for CPN and less than a month for chronic myocarditis/CM) stages of the disease, the incidence of PIFSI reaches $40 \%$. However, its ratio diminishes with further prolongation of the period of disease, while the contribution of PIFA and PIFASID increases in contrast.

(3) Time scale of progression and chronization of disease: In patients with a relapsing or rapidly progressing course (e.g., ICIIP, AIM), the contribution of PIFSI does not exceed 32-36\%, while that of autoimmune syndromes amounts to $80-100 \%$. It may thus be concluded that PIFSI is not only an outcome of the infectious disease, but, rather, is the major factor responsible for its lingering and chronic relapsing course. The role of progression and chronization of the disease is defined, in a large measure, by the form of PICIS or, rather, PIFA and PIFASID, which reflect the picture of post-infectious autoaggression.

\section{ASSOCIATIVE CORRELATION BETWEEN CLINICAL MANIFESTATIONS OF PICIS AND CRID: MODERN TRENDS IN THE PRE-EARLY DIAGNOSTICS}

Akhmedilova $\mathrm{KA}^{2}$, Agirov $\mathrm{MM}^{2}$, Tabaksoeva $\mathrm{JA}^{2}$, Cherepakhina $\mathrm{NE}^{1,3}$, Bodrova $\mathrm{TA}^{4}$, Shogenov $\mathrm{ZS}^{2}$, Poteshkina $\mathrm{NG}^{2}$, Suchkov $\mathrm{SV}^{1,2}$

${ }^{1}$ I.M. Sechenov First Moscow State Medical University (FMSMU), Moscow, Russia

${ }^{2}$ Russian State Medical University (RSMU), Moscow, Russia

${ }^{3}$ Federal Agency of Medical and Biological Problems under the Government of the Russian Federation, Moscow, Russia ${ }^{4}$ Department of Economy of Health Services, The Higher School of Economy, Moscow, Russia

The association between microbial landscapes (microbiome) and various immunopathological states with postinfectious clinical-and-immunological syndrome (PICIS) can be correlative or causal. In patients with chronically relapsing infectious diseases (CRID), syndromal forms of the immune-mediated disorders depend crucially on the stage of the inflammatory process occurring in the targeted organs or tissues and the overall chronization of the disease.

For example, early stages of CRID are concomitant with post-infectious secondary immunodeficiency syndrome (PIFSI) ( $>50 \%)$, whereas the incidence and thus the contribution of post-infectious autoimmune syndrome (PIFA) and autoimmune syndrome associated with postinfectious secondary immunodeficiency (PIFASID) do not exceed $20 \%$. At the subsequent stages, the clinical manifestations are different, viz., the incidence and thus the contribution of the autoimmunity rises dramatically: up to $50 \%$ at the intermediate stages (PIFA) and up to $60 \%$ at the final stage (PIFASID).

The correlation between the stage of CRID and the form of PICIS is also characterized by the involvement of an additional (the third) component, viz., a clinical form of CRID. Here are several analytical examples related to:

(1) a clinical form of CRID: in patients with primary pyelonephritis (PPNP) and infectious myocarditis (IM), PIFSI is detected in $75 \%$ of clinical cases, whereas in patients with secondary pyelonephrites (SPNP) and autoimmune myocardites (AIM) the contribution of PIFSI is notably reduced (to $25 \%$ ) giving way to the autoaggression (the incidence and thus the contribution of PIFA and PIFASID increases to $60 \%$ and $85 \%$, respectively);

(2) a stage of CRID: at the early (including preclinical ones) stages $(<3$ months for CPN and $<1$ month for myocarditis (M)), PIFSI is detected in $40 \%$ of cases; however, at the advanced stages of CRID its incidence reduced appreciably, while that of autoimmune syndromes increases in contrast;

(3) a rate of progression and chronization of CRID: in patients with relapsing or rapidly progressing CRID (e.g., ICIIP or AIM), the incidence and thus the contribution of PIFSI do not exceed 32-36\%, while the share of autoimmune syndromes reaches 80 $100 \%$. In such patients, persistent forms of chronic meningoencephalitis (e.g., ICIIP) or AIM associated with myocardial dystrophies are predominant.

These findings suggest that PIFSI is not only the outcome of the infectious process, but also the predictive sign to promote chronization of the disease and to represent a factor responsible for getting the clinical course chronic. Further progression and chronization of CRID are controlled by post-infectious autoaggression factors, such as PIFA and PIFASID which are thus also considered as predictive factors to monitor transformation of subclinical forms into the clinical ones. 
ANTITHYROID AUTOANTIBODY POPULATION AS A SOURCE OF ANTIBODY SPECIFICITY AND FUNCTIONALITY: CLINICAL SIGNIFICANCE OF THE PHENOMENON OF THE SEROLOGICAL ORCHESTRA IN MONITORING OF PATIENTS WITH GRAVES' DISEASE

Bogatyreva $Z I^{1}$, Andreeva $A V^{1}$, Suchkova $E N^{2}$, Notkins $A L^{3}$, Suchkov $S V^{4}$

${ }^{1}$ University of World Politics and Law, Faculty of Economy of Medical Technologies, Div of Endocrinology, Municipal Clinical Hospital №81, Moscow, Russia

${ }^{2}$ Department of Endocrinology, AGMA and AlexandroMariinskay Clinical Hospital, Astrakhan, Russia

${ }^{3}$ National Institutes of Health, Bethesda, MD, USA

${ }^{4}$ Department of Pathology, I.M.Sechenov First MMSU, Moscow, Russia

Introduction: The incidence and titers of serum autoantibodies (autoAT) depend on the clinical manifestations of autoimmune thyroid diseases and the expressiveness of the autoaggression. Among anti-thyroid autoAbs the following ones should be highlighted:

(1) canonical (anti-TG and anti-TPO) autoAbs and bispecific (TG and TPO simultaneously) autoAbs (antiTGPO autoAbs);

(2) autoAbs with the functional properties: anti-TSH-R autoAbs (TSH-R autoAbs), and autoAbs with proteolytic activity (Ab-proteases).

Anti-thyroid autoAbs are occurring in patients with thyroid dysfunction (hyper-or hypothyroidism: $55-90 \%$ of cases) and in euthyroid individuals ( $3-8 \%$ of cases).

Canonical autoAT: Anti-thyroid autoAbs of different specificities that dominat in Graves' disease ( $70 \%$ and more), are presented by canonical anti-TPO and anti-TG autoABs (60\%) and by anti-TGPO autoAbs (less $40 \%$ ). Dynamics of antithyroid autoAbs depends on the severity of the autoaggression and would result in the decrease prior to the early stages of the remission or to the burst at the exacerbation of the disease. Therefore, preclinical diagnostic protocol could assess the risks of Graves' disease to progress, and to thus implement the in-time therapeutic intervention to prevent the initiation of the preclinical stages of the disease.

Anti-TG autoAbs:Anti-TG autoAbs in Graves' patients occur with a frequency of $50 \%$.

Anti-TPO autoAbs:High-rate occurrence and serum titers of anti-TPO autoAbs illustrate featured complications and manifested forms of the disease ( $92-95 \%$ of cases), as well as subclinical Graves' disease (62-66\%).

Anti-TGRO autoAbs:In Graves' disease highly-enriched populations of anti-TGPO autoAbs have been found.

Clustering thyroid autoAbs: The most common triplet found in patients with manifested forms of Graves' disease is a serological panel of anti-TG-anti-TPO-anti-TGPO autoAbs as well as duplexes of anti-TPO-anti-TGPO autoAbs. No anti-TG-anti-TGPO duplexes have been identified in Graves' disease.

Functionally active autoAT: In patients with Graves' disease characterized by a high prevalence of functionally active autoAbs, in particular, the TSH-R and Ab-proteases have been dominated.

$T S H-R$ autoAbs:TSH-R autoAbs classified as functionally valuable Abs to mimic functions of the hormone and to trigger the development of the Graves' could be distinguished for two subtypes, i.e., TSH-stimulating (TCA) and TSH-blocking (TBA) autoAbs.

AT-proteases:The prevalence of AT-proteases in patients with Graves' disease was reached to $77 \%$ to include duplexes consisting of the TG-and TPO-Ab-specific proteases simultaneously. In the early stages of Graves' disease TPO-specific Ab-proteases are the only ones: they are $50 \%$ of patients with subclinical Graves' disease and $30 \%$ - with 0 goiter. The activity of Abproteases increases during the destruction of the thyroid (hypothyroidism): from stage to stage of the manifested complicated course the activity increased in two or more times. With a progression of the disease and the development of hyperthyroidism is also identified a tendency for the serological clustering.

Advanced serodiagnostic protocols: Currently there are no defined serological criteria to allow for putting timely diagnosis of Graves' disease. A way of solving the problem is the use of functionally active Abs (including Abproteases).A role in solving the problems to verify syndrome-like forms of the hyperthyroidism and/or to identify the preclinical stages of the clinical illness and to thus predict the latter would belong to Ab-proteases.

\section{HUMAN CHITINASE-LIKE PROTEINS: NEW BIOMARKER FAMILY}

Kzhyshkowska J, Riabov V, Wang N, Gratchev A

Medical Faculty Mannheim, University of Heidelberg, Germany

Mammalian chitinase-like proteins (CLP) are induced at sites of inflammation and in tumors. CLP are used as investigative biomarkers for various human pathologies including solid tumors (breast, colorectal, prostate cancers), and cardiovascular disorders (atherosclerosis and its consequences). The common structural feature of mammalian chitinases and chitinase-like proteins is the presence of conserved Glyco 18 domain. Function of chitinases in lower life forms is a host defense against chitin-containing organisms. There are 6 Glyco_18 domain containing proteins identified in human up to date. Most recently, SI-CLP has 
been identified by us as a last chitinase-like protein which secretion is regulated by receptor stabilin-1 in macrophages. Human CLP comprise YKL-39, YKL-40, and SI-CLP. CLP lack critical amino acids within the catalytic site and do not exhibit enzymatic activity. Their biological activity is defined by specific interactions mediated by enzymatically silent Glyco_18 domain. Human CLP are secreted into the extracellular space, and can be detected in tissues and in blood circulation.

YKL-40 is the best investigated human CLP regarding its association with various disorders. Circulating YKL-40 can be detected in human serum or plasma using RIA or ELISA. Increased concentrations of YKL-40 are associated with disorders characterized by different types of inflammation including asthma, arthritis, and atherosclerosis. Elevated levels of YKL-40 were found in patients with various solid tumors including breast cancer, colorectal cancer, ovarian cancer, metastatic renal and prostate cancer and malignant melanoma. High levels of YKL-40 are associated with poor prognosis and rapid metastatic process. Levels of YKL-40 reflect difference in biology of cancer cells themselves as well as activation tumor-associated macrophages.

YKL-39 was identified as an abundantly secreted protein in primary culture of human articular chondrocytes. YKL-39 is currently recognized as a biomarker for the activation of chondrocytes and osteoarthritis progression. For a long time it was believed that macrophages do not secrete YKL-39. However recently we demonstrated that key regulatory factor of development, tumor progression and pathogenesis of arthrosclerosis, TGFbeta, strongly stimulates YKL-39 expression in macrophages.

SI-CLP (stabilin-interacting chitinase-like protein) is the most recently identified by us human CLP. Th2 cytokine IL4 and by dexamethasone induce expression of SI-CLP in macrophages. We developed rat $\mathrm{mAb} 1 \mathrm{C} 11$ that recognized SI-CLP in the cellular fraction of bronchoalveolar lavage obtained from patients with chronic inflammatory disorders of the respiratory tract and in PBLs from these patients. SI-CLP is the only CLP that is up-regulated by glucocorticoids. The highest expression level of SI-CLP was found by us in a patient with sarcoidosis undergoing glucocorticoid therapy. We observed strong differences in effect of dexamethasone on SI-CLP production in macrophages from different healthy individuals. Thus, SI-CLP is a promising marker for the individual response to glucocorticoids and prediction of side effects of corticoid treatment. Our most recent data indicate that SI-CLP is expressed in human colon carcinoma cells, but not in breast or lung carcinoma cells indicating the perspective for using SI-CLP as a cancer biomarker.
MACROPHAGE-BASED TEST SYSTEM FOR THE ANALYSIS OF IMMUNE STATUS AND HIDDEN IMMUNOPATHOLOGY DIAGNOSTICS

Gratchev $A^{1}$, Orekhov $A N^{2}$, Kzhyshkowska $J^{l}$

${ }^{1}$ Medical Faculty Mannheim, University of Heidelberg, Theodor-Kutzer Ufer 1-3, 68167 Mannheim, Germany

${ }^{2}$ Institute of General Pathology and Pathophysiology, Russian Academy of Medical Sciences, Moscow, Russia

Hidden immunopathologies play an important role in the development of such life threatening diseases as atherosclerosis, including its potential consequences (heart attack and stroke), cancer and dementia. Several cellular components are responsible for the regulation of the activity of immune system. The key regulatory role in this process is played by monocytes and macrophages. Being distributed in all tissues and organs of the human body macrophages control its condition and guarantee timely and effective reaction to damage, pathogen intrusion or transformed cell appearance. After effective inflammatory reaction and removal of the danger, macrophages initiate healing processes and restore tissue homeostasis. Thus malfunction of macrophages underlines most immune disorders.

We have reported that macrophages may adapt their phenotype to changing microenvironment. According to their pro- and anti-inflammatory activity macrophages can be classified as type 1 (M1) and type 2 (M2) respectively. Various pro- and anti-inflammatory stimuli are responsible for high heterogeneity of macrophage phenotype in each class. Type 1 macrophages produce pro-inflammatory cytokines and express Fc-gamma receptors on the cell surface. Type 2 macrophages produce anti-inflammatory cytokines and chemokines and express various scavenger receptors. The mechanism of macrophage activation is highly complex and includes several signalling cascades, vesicular trafficking and interaction of these two processes. In case of immune system dysfunction blood monocytes that have to be in inactive state gain properties of activated macrophages and change their surface markers. The analysis of a balance between pro- and anti-inflammatory activities of primary human monocyte-derived macrophages is an attractive possibility for identification of hidden immunopathologies. We have developed a test system for the complex analysis of activation state of primary human monocytes that provides highly valuable information about the presence of immune system malfunction before any clinical manifestation of a disease. This system can be also used for the development of immunocorrecting, anti-atherosclerotic and anti-cancer drugs. 